to the accumulation of iron oxide in the nodal macrophages, whereas metastatic nodes show either a heterogeneous decrease or no decrease in signal, since the normal macrophages are replaced with cancer cells.

By comparing the MRI results with histologic findings for 172 dissected nodes, the authors showed that the sensitivity of ferumoxtran-10enhanced MRI was significantly better than that obtained using precontrast images alone (96\% vs $76 \%, P<0.01)$ and that the negative predictive value was also significantly improved (98\% vs $91 \%, P<0.01$ ).

Noting that ferumoxtran-10-enhanced MRI could be used to identify metastases even in normal-sized nodes, the authors conclude that the technique allows superior preoperative nodal staging in patients with bladder cancer, compared with standard, nonenhanced MRI.

Original article Deserno WMLLG et al. (2004) Advances in preoperative nodal staging for bladder cancer. Radiology 233: 449-456

\section{Four weeks of tadalafil for ED improves endothelial function}

Endothelial impairment is an etiological factor common to cardiovascular disease and erectile dysfunction (ED). As the oral phosphodiesterase-5 inhibitor sildenafil (Viagra ${ }^{\circledR}$; Pfizer, Walton-on-theHill, UK) has been shown to improve endothelial function in men with ED, Rosano et al. tested the hypothesis that its long-acting counterpart tadalafil (Cialis ${ }^{\circledR}$; Lilly, Basingstoke, UK) would have a similar effect in men at increased risk of cardiovascular disease.

Thirty-two men with a 10-year cardiovascular risk exceeding $20 \%$, with or without ED, were randomized in a double-blind fashion to either $20 \mathrm{mg}$ tadalafil every second day or to placebo. At the end of the 4-week study period, flow-mediated dilatation (FMD) of the brachial artery had improved significantly to $9.3 \%$ in the tadalafil group. No change in FMD was detected in those men that had received placebo. Interestingly, the improvement of FMD in the tadalafil group was maintained for at least 2 weeks after discontinuation of therapy. This sustained increase in FMD was inversely correlated with plasma levels of endothelin-1, a peptide hormone associated with vascular disease. ED status had no effect on outcomes, and only two men receiving the study drug reported adverse events.

The authors have shown that tadalafil has beneficial effects on endothelial function that are maintained following cessation of therapy. Together with the fact that this drug can be used chronically for ED, rather than requiring 'on demand' administration like sildenafil, these new findings make tadalafil worthy of further investigation by both urologists and cardiologists.

Original article Rosano GMC et al. (2005) Chronic treatment with tadalafil improves endothelial function in men with increased cardiovascular risk. Eur Urol 47: 214-222 